\title{
Entropy of controlled invariant subspaces
}

\author{
Fritz Colonius $^{1, *}$ and Uwe Helmke ${ }^{2, * *}$ \\ ${ }^{1}$ Institut für Mathematik, Universität Augsburg, Universitätsstraße, 86135 Augsburg, Germany \\ 2 Institut für Mathematik, Universität Würzburg, Am Hubland, 97074 Würzburg, Germany
}

Key words Invariance entropy, topological entropy, (A,B)-invariant subspaces.

For continuous-time linear control systems invariance entropy of controlled invariant subspaces is introduced. It is shown that it coincides with a variant of topological entropy for linear flows which we call subspace entropy. Using this characterization, upper bounds in terms of eigenvalues of an induced flow are derived. Under additional assumptions (diagonalizability, single inputs) these bounds are improved

\section{Introduction}

Control of systems through digital communication channels and estimation of the required data rates have been considered since more than twenty years. The main motivation for this circle of ideas comes from the increasing needs of controlling systems with communication constraints, i.e. for systems where the state passes through a communication channel and may thus be delayed, corrupted by noise or even lost, and thus in any case may not be fully available to the controller. Early contributions to the field are, among others, due to Wong and Brockett [33,34]. More recently, problems in networked control system have been treated, see, e.g., Gupta et al. [15], or Carli and Bullo [5] for quantized coordination algorithms for robots. In [23], Nair et al. introduced ideas from topological dynamics into this field (see Katok and Hasselblatt [16] for an authoritative presentation of the mathematical theory of dynamical systems.) They introduced and studied the notions of topological feedback entropy and symbolic controllers for the problem of stabilizing a discrete-time system with communication constraints at an equilibrium.

The theoretical analysis of linear systems, controlled over communication channels, profits from the combination of concepts from linear systems theory and information theory alike. In particular, the information theoretic notion of entropy plays a central role here as it leads to effective quantitative bounds on data rates necessary for feedback stabilization. Up to now, such information-based investigations have not been connected with the constructions and the insights provided by classical geometric control theory which started with the early work of Basile and Marro [2] and Wonham [32]. We also refer to the textbook Basile and Marro [3] and to Trentelman, Stoorvogel, and Hautus [27] as well as the recent tutorial Marro et al. [21]. Controlled invariant subspaces form a cornerstone of geometric control theory and play a crucial role in understanding controller design problems such as disturbance decoupling, filtering, robust observer design, and high gain state feedback. We therefore expect a similar impact on the analysis of linear systems that are controlled over communication channels.

In this paper we begin an investigation of how geometric control design via controlled invariant subspaces is affected by entropy estimates and associated data rate constraints. As a starting point for such an investigation, we associate to any $(A, B)$-invariant subspace $V$ of a linear control system a state space similarity invariant, called the invariance entropy of $V$, that measures how difficult it is, using open loop controls, to keep the system in $V$. It is defined by the exponential growth rate of the number of controls necessary to keep the system in an arbitrarily small $\varepsilon$-neighborhood of $V$. We show that the invariance entropy is finite for any $(A, B)$-invariant subspace and derive upper bounds in terms of the sum of the eigenvalues of $A$ with positive real part. Sharper upper bounds are derived for specific classes of linear systems. In turned out that all constructions can be naturally extended for the larger class of almost $(A, B)$-invariant subspaces, hence the latter are considered throughout this paper (cf. Remark 4.2.) Our approach follows and partially extends that by Colonius and Kawan [8], where an entropy-like notion was proposed for controlled invariance of compact subsets with nonvoid interior in the state space of general control systems. Further results are given in Kawan [17-19] and Colonius and Kawan [8]. This entropy notion may be regarded as a lower bound for the minimum data rate (take the logarithm with base 2 instead of the

\footnotetext{
* Corresponding author E-mail: fritz.colonius@math.uni-augsburg.de

** E-mail: helmke@mathematik.uni-wuerzburg.de
} 
natural logarithm used, for convenience, in the present paper.) More explicit relations to data rates and to the topological feedback entropy introduced in [23] are also given in Kawan's doctoral thesis [20], see also Colonius [6,7] for a discussion of the required bit rates. We defer applications of the estimates given here to a later paper.

The contents of this paper are as follows: Sect. 2 recalls basic facts on $(A, B)$-invariant and on almost $(A, B)$-invariant subspaces. Section 3 introduces a subspace entropy for homogeneous autonomous linear differential equations. Section 4 presents the main concept of the paper, invariance entropy of almost $(A, B)$-invariant subspaces, and shows that it coincides with the subspace entropy of the uncontrolled system. Final Section 5 provides an upper bound for invariance entropy in terms of eigenvalues. In special cases, sharper upper bounds can be shown.

Notation. The distance of a point $x$ in a normed vector space to a closed subset $M$ is defined by $\operatorname{dist}(x, M):=$ $\inf _{y \in M}\|x-y\|$.

\section{Preliminaries on controlled-invariant subspaces}

The purpose of this section is to summarize some well-known definitions and facts from geometric control theory, i.e., controlled and almost controlled invariant subspaces. The notion of controlled invariant or, equivalently, $(A, B)$-invariant subspaces was introduced by Basile and Marro [2], and Wonham [32]. The concept of almost controlled invariant subspaces was first introduced by Willems [30,31]; we also refer to the doctoral theses by Trentelman [26] and Trumpf [28] for a useful summary of basic definitions and facts.

Consider linear control systems in state space form

$$
\dot{x}(t)=A x(t)+B u(t)
$$

with matrices $A \in \mathbb{R}^{n \times n}$ and $B \in \mathbb{R}^{n \times m}$. The solutions $\varphi(t, x, u), t \in \mathbb{R}$, of (2.1) with initial condition $\varphi(0, x, u)=x$ are given by the variations-of-constants formula:

$$
\varphi(t, x, u)=\mathrm{e}^{A t} x+\int_{0}^{t} \mathrm{e}^{A(t-s)} B u(s) d s .
$$

Recall that a subspace $V$ is called $(A, B)$-invariant, if for all $x \in V$ there is $u \in \mathbb{R}^{m}$ with $A x+B u \in V$. Equivalently, there is a matrix $F \in \mathbb{R}^{m \times n}$, a so-called friend of $V$, such that for $A_{F}:=A+B F$

$$
A_{F} V \subset V
$$

This can be seen by choosing for a basis $x_{1}, \ldots, x_{k}$ of $V$ control values $u_{1}, \ldots, u_{k} \in \mathbb{R}^{m}$ with $A x_{i}+B u_{i} \in V$. Then extend this to a basis of $\mathbb{R}^{n}$ and define a linear map $F$ by

$$
F x_{i}=u_{i} \text { for } i=1, \ldots, k \text {, and } F \text { arbitrary outside } V .
$$

This also shows that $V$ is $(A, B)$-invariant if and only if it is controlled invariant, i.e., for every $x \in V$ there is an open loop continuous control function $u:[0, \infty) \rightarrow \mathbb{R}^{m}$ with $\varphi(t, x, u) \in V$ for all $t \geq 0$. In fact, differentiating the solution one finds

$$
V \ni \frac{d}{d t} \varphi(0, x, u)=A x+B u(0)
$$

Conversely, define for $x \in V$ a control by $u(t)=F \mathrm{e}^{(A+B F) t} x, t \geq 0$.

More generally, a linear subspace $V$ is called almost $(A, B)$-invariant, if for any $x \in V$ and any $\varepsilon>0$ there exists a control function $u(\cdot)$ such that for all $t \geq 0$

$$
\operatorname{dist}(\varphi(t, x, u), V)=\inf _{y \in V}\|\varphi(t, x, u)-y\|<\varepsilon .
$$

Almost $(A, B)$-invariant subspaces are of interest to study subspaces invariant under high gain state feedback. Thus, in general, almost $(A, B)$-invariant subspaces cannot be made invariant under state feedback, so there is no friend, but they can be made almost-invariant in the sense that for every $x \in V$ and any $\varepsilon>0$ there exists a feedback $F$ such that for all $t \geq 0$

$$
\operatorname{dist}\left(\mathrm{e}^{A_{F} t} x, V\right)<\varepsilon,
$$


cf. [26]. The basic theory of almost $(A, B)$-invariant subspaces has been developed by Willems in [30, 31], including a discussion of the differences to $(A, B)$-invariant subspaces and the feedback characterization given above. Since the 'almost' property is of no relevance in the present paper, we do not repeat these classical results here. A reader not familiar with this theory may just restrict attention to $(A, B)$-invariant subspaces.

In order to derive explicit estimates for the entropy of controlled invariant subspaces it is useful to have explicit parametrizations of the class of all controlled invariant subspaces. This is a difficult task and we refer to e.g. [13], [28] for further information. Certain types of subspaces are of special interest here. A controlled invariant subspace $V$ is called coasting, if $V \cap \operatorname{Im} B=\{0\}$. Equivalently, $\{0\}$ is the largest controllability subspace contained in $V$. Any controlled invariant subspace of a controllable single-input system is coasting.

For an $(A, B)$-invariant subspace $V$ and a friend $F \in \mathbb{R}^{m \times n}$, the subspace $V$ is $A_{F}$-invariant. The restriction $(\bar{A}, \bar{B})$ is given by $\bar{A}:=A_{F} \mid V$, the restriction of $A_{F}$ to $V$, and $\bar{B}:=B \mid B^{-1} V: B^{-1} V \rightarrow V$, the restriction of $B$ to the linear subspace $B^{-1} V:=\left\{u \in \mathbb{R}^{m} \mid B u \in V\right\}$. The co-restriction $(\tilde{A}, \tilde{B})$ is given by $\tilde{A}: \mathbb{R}^{n} / V \rightarrow \mathbb{R}^{n} / V$, the map induced by $A_{F}$, and $\tilde{B}: \mathbb{R}^{m} / B^{-1} V \rightarrow \mathbb{R}^{n} / V$ is the map induced by $B$. Note, that the co-restriction $(\tilde{A}, \tilde{B})$ is controllable, whenever $(A, B)$ is controllable, while the restriction is controllable only for a controllability subspace. Note also, that $\bar{B}$ and $\tilde{B}$ are both full column rank if $B$ has full column rank. Of course, the co-restriction may well depend upon the choice of a friend $F$, so there are in fact many possible co-restrictions and not just one. However, the controllability indices of the co-restrictions are all the same. It is thus a remarkable but simple fact, that for any coasting subspace $V$, the co-restriction is uniquely defined and independent of $F$.

Following the notation from Wonham's book [32], we denote by $\langle A \mid V\rangle$ the smallest $A$-invariant subspace containing $V$. Furthermore, the largest $A$-invariant subspace that is contained in $V$, is denoted by $\mathfrak{I}^{*}(A ; V)$. Generically, one expects $\langle A \mid V\rangle=\mathbb{R}^{n}$ and $\mathfrak{I}^{*}(A ; V)=\{0\}$, but one can be more specific. If $V$ is an $(A, B)$-invariant subspace that is coasting, then there is some a-priori information about the dimensions of the bounding subspaces. For simplicity, we focus on the single input case, i.e. $m=1$.

Lemma 2.1. Let $(A, b)$ be controllable and let $V$ be any $(A, b)$-invariant subspace that is not A-invariant. Then every $A$-invariant subspace $W \supset V$ satisfies $W=\mathbb{R}^{n}$. Any generic $(A, b)$-invariant subspace satisfies $\mathfrak{I}^{*}(A ; V)=\{0\}$ and $\langle A \mid V\rangle=\mathbb{R}^{n}$.

Pro of. Note, that in the single input case only, every $(A, b)$-invariant subspace is automatically coasting. By duality, it suffices to show for single-output systems $(c, A)$ that $\langle A \mid V\rangle=\mathbb{R}^{n}$, for any tight $(c, A)$-invariant subspace $V$ that is not $A$ invariant. In order to show this we apply the theory of polynomial models; cf. Fuhrmann [11], Fuhrmann and Willems [12]. Let $q(z)=\operatorname{det}(z I-A)$ denote the characteristic polynomial and $X_{q}$ denote the associated polynomial model. Thus, $X_{q}$ denotes the set of polynomials of degree $<n$ with the module structure given by multiplication modulo $q$. The (tight) conditioned invariant subspaces of codimension $d$ then uniquely correspond to the intersection

$$
V=X_{q} \cap t(z) \mathbb{R}[z]
$$

via a unique monic polynomial $t$ of degree $d$; see Fuhrmann and Helmke [13]. Let $V_{*}\left(V^{*}\right)$ denote the largest (smallest) shift invariant subspace of $X_{d}$ contained in $V$ (containing $V$ ). In this polynomial framework, the $A$-invariant subspaces are of the form $q_{1} X_{q_{2}} \subset X_{q}$, for any factorization $q=q_{1} q_{2}$. Thus $q_{1} X_{q_{2}} \subset X_{q} \cap t(z) \mathbb{R}[z]$ if and only if $t$ divides $q_{1}$. In particular, $t$ must then divide $q$, i.e. $V$ must be an invariant subspace. Now assume that $V$ is not an invariant subspace (for the shift), i.e. $t$ does not divide $q$. Then $V$ does not contain any nontrivial invariant subspace. Applying duality, this implies the lemma. But more can be said. Factor $t=q_{1} a$ with $q_{1}$ a polynomial of degree $r$ dividing $q, q=q_{1} q_{2}$, and $a$ a polynomial that is coprime to $q$. Then $q_{1} X_{q_{2}}$ is the smallest invariant subspace containing $V$. The codimension of this subspace is thus deg gcd $\{q, t\}$. In particular, if $q$ and $t$ are coprime, then $V_{*}=\{0\}$ and $V^{*}=X_{q}$.

\section{Subspace entropy of linear flows}

In this section we define for a linear autonomous differential equation the entropy of a linear subspace $V \subset \mathbb{R}^{n}$. It is a suitable adaptation of the well-known topological entropy of the flow associated with an autonomous linear differential equation, see e.g. Walters $[29, \S 8.4]$. Later we will use it for the uncontrolled system $\dot{x}=A x$ and relate it to the entropy of controlled invariant subspaces (cf. Definition 4.3). We would like to emphasize that the open loop control system (2.1) does not define a flow, since the control functions $u(\cdot)$ are time-dependent, and hence it is not covered by this definition.

Let $V$ be a linear subspace of $\mathbb{R}^{n}$. For a linear map $A: \mathbb{R}^{n} \rightarrow \mathbb{R}^{n}$, let $\Phi: \mathbb{R} \times \mathbb{R}^{n} \rightarrow \mathbb{R}^{n}, \Phi(t, x):=\mathrm{e}^{A t} x, t \in \mathbb{R}, x \in$ $\mathbb{R}^{n}$ be the induced flow (actually, throughout this paper, only the semiflow defined for $t \geq 0$ will be relevant.). For any compact subset $K \subset V$ and for given $T, \varepsilon>0$ we call $R \subset K$ a $(T, \varepsilon, K, V ; \Phi)$-spanning set, if for all $x \in K$ there exists 
$y \in R$ with

$$
\max _{0 \leq t \leq T} \operatorname{dist}\left(\mathrm{e}^{t A}(x-y), V\right)<\varepsilon
$$

Let $r(T, \varepsilon, K, V ; \Phi)$ denote the minimal cardinality of a $(T, \varepsilon, K, V ; \Phi)$-spanning set. Observe that $K$ is always a $(T, \varepsilon, K, V ; \Phi)$-spanning set. By compactness of $K$ and continuous dependence on the initial value, this implies that a finite $(T, \varepsilon, K, V ; \Phi)$-spanning set exists. Similarly, we call $S \subset K$ a $(T, \varepsilon, K, V ; \Phi)$-separated set, if for all $x \neq y$ in $S$

$$
\max _{0 \leq t \leq T} \operatorname{dist}\left(\mathrm{e}^{t A}(x-y), V\right) \geq \varepsilon
$$

The maximal cardinality of such a set is denoted by $s(T, \varepsilon, K, V ; \Phi)$. Note that the points $y$ in $R$ (and in $S$ ) will, in general, not lead to solutions $\mathrm{e}^{A t} y$ which remain for all $t \geq 0$ in the $\varepsilon$-neighborhood of $V$. In the following, we will omit some of the arguments in the notions above and in similar ones defined below, if they are clear from the context.

Definition 3.1. Let $A$ be a linear map on $\mathbb{R}^{n}$ with associated flow $\Phi$ and consider a subspace $V$ of $\mathbb{R}^{n}$. For a compact subset $K \subset V$, we consider the exponential growth rate of $r(T, \varepsilon, K, V ; \Phi)$ and set

$$
\begin{aligned}
h_{\text {span }}(\varepsilon, K, V ; \Phi) & :=\limsup _{T \rightarrow \infty} \frac{1}{T} \ln r(T, \varepsilon, K, V ; \Phi), \\
h_{\text {span }}(K, V ; \Phi) & :=\lim _{\varepsilon \searrow 0} h_{\text {span }}(\varepsilon, K, V ; \Phi),
\end{aligned}
$$

and define the entropy of $\Phi$ with respect to $V$ by

$$
h(V ; \Phi):=\sup _{K} h_{\text {span }}(K, V ; \Phi),
$$

where the supremum is taken over all compact subsets $K \subset V$.

Analogously, an entropy of $\Phi$ with respect to $V$ can be defined via maximal separated sets.

As usual in the context of topological entropy, one sees that, by monotonicity, the limit for $\varepsilon \searrow 0$ exists (it might be infinite). Since all norms on a finite dimensional vector space are equivalent, the entropy does not depend on the norm used in (3.1). One easily sees that the subspace entropy $h(V ; \Phi)$ is invariant under state space similarity, i.e., $h\left(S V ; S \Phi S^{-1}\right)=$ $h(V ; \Phi)$ for $S$ in the set $G L(n, \mathbb{R})$ of invertible $n \times n$-matrices; here $S \Phi(t, \cdot) S^{-1}=S \mathrm{e}^{A t} S^{-1}=\mathrm{e}^{S A S^{-1} t}, t \geq 0$.

Remark 3.2. If we choose $V=\{0\}$ condition (3.1) is trivial, since only $K=\{0\}$ is allowed; furthermore, if we choose $V=\mathbb{R}^{n}$, the distance in (3.1) is always equal to zero. In particular, the subspace entropy does not recover the usual definition of topological entropy for the linear flow $\Phi(t, x)=\mathrm{e}^{A t} x$, where, for a compact subset $K \subset \mathbb{R}^{n}$, spanning sets are defined as minimal sets $R$ such that for all $x \in K$ there exists $y \in R$ with

$$
\max _{0 \leq t \leq T} \mathrm{~d}\left(\mathrm{e}^{t A} x, \mathrm{e}^{A t} y\right)=\max _{0 \leq t \leq T}\left\|\mathrm{e}^{t A}(x-y)\right\|<\varepsilon
$$

Equivalently, one can use the number $s_{\text {top }}(T, \varepsilon, K ; \Phi)$ of elements in maximal separated sets. For the topological entropy of linear flows, Bowen [4] could show

$$
h_{\text {top }}(\Phi):=\sup _{K} h_{\text {top }}(K ; \Phi)=\sum_{i=1}^{n} \max \left(0, \operatorname{Re} \lambda_{i}\right) \text {, }
$$

where $\lambda_{1}, \ldots, \lambda_{n}$ denote the eigenvalues of $A$; see also Walters [29, Theorem 8.14] and Matveev and Savkin [22, Theorem 2.4.2] for proofs.

The definitions via separated and spanning sets coincide, which easily follows from the next proposition (cf. Robinson [25, Lemma VIII.1.10] for similar arguments).

Proposition 3.3. Let $K \subset V$ be compact and fix $T, \varepsilon>0$. Then

$$
s(T, 2 \varepsilon, K, V ; \Phi) \leq r(T, \varepsilon, K, V ; \Phi) \leq s(T, \varepsilon, K, V ; \Phi) .
$$

Proof. $\quad$ Let $S \subset K$ be a maximal $(T, \varepsilon)$-separated set and let $x \in K$. By maximality of $S$, there is some $y \in S$ such that

$$
\max _{0 \leq t \leq T} \operatorname{dist}\left(\mathrm{e}^{t A}(x-y), V\right)<\varepsilon .
$$


Therefore $S$ is $(T, \varepsilon)$-spanning showing the second inequality. For the first one, consider a maximal $(T, 2 \varepsilon)$-separated set $S$ and a minimal $(T, \varepsilon)$-spanning set $R$. We define a map $H: S \rightarrow R$ in the following way: For $x \in S$ there is $y:=H(x) \in R$ with $\operatorname{dist}\left(\mathrm{e}^{t A}(x-y), V\right)<\varepsilon$ for all $t \in[0, T]$. If $H\left(x_{1}\right)=H\left(x_{2}\right)=y$, then

$$
\max _{0 \leq t \leq T} \operatorname{dist}\left(\mathrm{e}^{t A}\left(x_{1}-x_{2}\right), V\right) \leq \max _{0 \leq t \leq T} \operatorname{dist}\left(\mathrm{e}^{t A}\left(x_{1}-y\right), V\right)+\max _{0 \leq t \leq T} \operatorname{dist}\left(\mathrm{e}^{t A}\left(x_{2}-y\right), V\right)<2 \varepsilon .
$$

Thus $x_{1}=x_{2}$ follows. This shows that $H$ is injective, and hence $r(T, \varepsilon, K, V ; \Phi) \geq s(T, 2 \varepsilon, K, V ; \Phi)$.

Although this will not be used in the sequel, we describe the behavior of this entropy notion under a semiconjugacy to the induced flow on a quotient space $\mathbb{R}^{n} / W$, where the norm is given by $\|x+W\|:=\inf \{\|x+w\|, w \in W\}, x \in \mathbb{R}^{n}$.

Proposition 3.4. Let $W$ be an A-invariant subspace for a linear map $A$ on $\mathbb{R}^{n}$. Then, for a subspace $V$ of $\mathbb{R}^{n}$ the entropies of the induced flows $\Phi(t, x)=\mathrm{e}^{A t} x$ on $\mathbb{R}^{n}$ and $\bar{\Phi}(t, \bar{x})$ on the quotient space $\mathbb{R}^{n} / W$, respectively, satisfy

$$
h(V ; \Phi) \geq h(V / W ; \bar{\Phi}) .
$$

Proof. Let $K \subset V$ be compact and for $T, \varepsilon>0$ consider a $(T, \varepsilon, K, V ; \Phi)$-spanning set $R \subset K$. Denote the projection of $\mathbb{R}^{n}$ to $\mathbb{R}^{n} / W$ by $\pi$, hence $\pi V=V / W$. Then the set $\pi R$ is a $(T, \varepsilon, \pi K, \pi V ; \bar{\Phi})$-spanning set. In fact, let $R=\left\{x_{1}, \ldots, x_{\ell}\right\}$ and consider $\pi x \in \pi K$ for some element $x \in K$. Then there exists $x_{j} \in R$ with

$$
\max _{0 \leq t \leq T} \operatorname{dist}\left(\mathrm{e}^{t A}\left(x-x_{j}\right), V\right)<\varepsilon
$$

Denoting the map induced by $A$ on $\mathbb{R}^{n} / W$ by $\bar{A}$ one finds for all $t \in[0, T]$

$$
\begin{aligned}
\operatorname{dist}\left(\mathrm{e}^{t \bar{A}}\left(\pi x-\pi x_{j}\right), \pi V\right) & =\inf _{z \in V}\left\|\mathrm{e}^{t \bar{A}}\left(\pi x-\pi x_{j}\right)-\pi z\right\| \\
& =\inf _{z \in V, w \in W}\left\|\mathrm{e}^{t A}\left(x-x_{j}\right)-z-w\right\| \\
& \leq \operatorname{dist}\left(\mathrm{e}^{t A}\left(x-x_{j}\right), V\right) \\
& <\varepsilon .
\end{aligned}
$$

It follows that the minimal cardinality of a $(T, \varepsilon, K, V ; \Phi)$-spanning set is greater than or equal to the minimal cardinality of a $(T, \varepsilon, \pi K, \pi V ; \bar{\Phi})$-spanning set. Then take the limit superior for $T \rightarrow \infty$ and let $\varepsilon$ tend to 0 . Finally, observe that for every compact set $K_{1} \subset V / W$ there is a compact set $K \subset V$ with $\pi K=K_{1}$. Hence taking the supremum over all compact $K_{1} \subset V / W$ one obtains the assertion.

\section{Entropy for controlled invariant subspaces}

We now introduce the central notion of this paper, invariance entropy for almost $(A, B)$-invariant subspaces of linear control system (2.1) on $\mathbb{R}^{n}$ and relate it to the subspace entropy defined in the previous section.

In the following, we consider a fixed almost $(A, B)$-invariant subspace $V$ of $\mathbb{R}^{n}$ with $\operatorname{dim} V=d$. Furthermore, we admit arbitrary controls in the space $C\left([0, \infty), \mathbb{R}^{m}\right)$ of continuous functions $u:[0, \infty) \rightarrow \mathbb{R}^{m}$.

Definition 4.1. For a compact subset $K \subset V$ and for given $T, \varepsilon>0$ we call a set $\mathcal{R} \subset C\left([0, \infty), \mathbb{R}^{m}\right)$ of control functions $(T, \varepsilon, K, V)$-spanning if for all $x_{0} \in K$ there is $u \in \mathcal{R}$ with

$$
\operatorname{dist}\left(\varphi\left(t, x_{0}, u\right), V\right)<\varepsilon \text { for all } t \in[0, T] .
$$

By $r_{\text {inv }}(T, \varepsilon, K, V)$ we denote the minimal cardinality of a $(T, \varepsilon, K, V)$-spanning set. If no finite $(T, \varepsilon, K, V)$-spanning set exists, we set $r_{\text {inv }}(T, \varepsilon, K, V)=\infty$.

In other words: we require for a $(T, \varepsilon, K, V)$-spanning set $\mathcal{R}$ that, for every initial value in $K$, there is a control in $\mathcal{R}$ such that up to time $T$ the trajectory remains in the $\varepsilon$-neighborhood of $V$. Note that, in contrast to the definition of the subspace entropy for flows, Definition 3.1, here a number of control functions is counted, not a number of initial values. Hence this is a notion which is intrinsic to control systems. We also note that the definition above differs from earlier ones used for invariance entropy (cf. [8,9]) by the fact, that the set $V$ whose invariance is studied here, is not compact and that the controls are unrestricted.

The following observation shows that there are always finite $(T, \varepsilon, K, V)$-spanning sets of control functions. 
Remark 4.2. Let $K \subset V$ be compact and $\varepsilon, T>0$. By almost $(A, B)$-invariance of $V$ there is for every $x \in K \subset V$ a control function $u$ with $\operatorname{dist}(\varphi(t, x, u), V)<\varepsilon$ for all $t \geq 0$. Hence, using continuous dependence on initial values and compactness of $K$, one finds finitely many controls $u_{1}, \ldots, u_{r}$ such that for every $x \in K$ there is $u_{j}$ with $\operatorname{dist}\left(\varphi\left(t, x, u_{j}\right), V\right)<\varepsilon$ for all $t \in[0, T]$. Hence $r_{\mathrm{inv}}(T, \varepsilon, K, V)<\infty$. This is the reason why we consider in the following almost $(A, B)$-invariant subspaces, not just $(A, B)$-invariant subspaces. It also seems, that the class of almost $(A, B)$-invariant subspaces is the largest class of subspaces such that for every compact subset $K$ and every $T, \varepsilon>0$ this number is finite.

Now we consider the exponential growth rate of $r_{\mathrm{inv}}(T, \varepsilon, K, V)$ for $T \rightarrow \infty$ and let $\varepsilon \rightarrow 0$. The resulting invariance entropy is the main subject of the present paper.

Definition 4.3. Let $V$ be an almost $(A, B)$-invariant subspace. Then, for a compact subset $K \subset V$, the invariance entropy $h_{\mathrm{inv}}(K, V)$ is defined by

$$
h_{\mathrm{inv}}(\varepsilon, K, V):=\limsup _{T \rightarrow \infty} \frac{1}{T} \ln r_{\mathrm{inv}}(T, \varepsilon, K, V), h_{\mathrm{inv}}(K, V):=\lim _{\varepsilon \searrow 0} h_{\mathrm{inv}}(\varepsilon, K, V) .
$$

Finally, the invariance entropy of $V$ is defined by

$$
h_{\text {inv }}(V ; A, B):=\sup _{K} h_{\text {inv }}(K, V) \text {, }
$$

where the supremum is taken over all compact subsets $K \subset V$.

In the sequel, we will always use for a given underlying system $(A, B)$ the shorthand notation $h_{\text {inv }}(V)$ for $h_{\text {inv }}(V ; A, B)$. Note that $h_{\text {inv }}\left(\varepsilon_{1}, K, V\right) \leq h_{\text {inv }}\left(\varepsilon_{2}, K, V\right)$ for $\varepsilon_{2} \leq \varepsilon_{1}$. Hence the limit for $\varepsilon \rightarrow 0$ exists (it might be infinite.) Since all norms on finite dimensional vector spaces are equivalent, the invariance entropy of $V$ is independent of the chosen norm. We will show later that every almost $(A, B)$-invariant subspace has finite invariance entropy. It is clear by inspection, that, as the subspace entropy $h(V ; \Phi)$, also the invariance entropy $h_{\text {inv }}(V)$ is invariant under state space similarity; i.e. $h_{\text {inv }}\left(S V ; S A S^{-1}, S B\right)=h_{\text {inv }}(V ; A, B)$ for $S \in G L(n, \mathbb{R})$.

We are interested in the problem to keep the system in the subspace $V$ for all $t \geq 0$. Then the exponential growth rate of the required number of control functions will give information on the difficulty of this task. Since, also on finite intervals, the number of controls for achieving invariance need not be finite, an appropriate mathematical formulation requires a slightly relaxed notion, which allows for arbitrarily small deviations from the subspace $V$. The motivation to consider open-loop controls in this context comes, in particular, from model predictive control (see, e.g., the collection Allgöwer and Zheng [1] and Grüne and Pannek [14]), where optimal open-loop controls are computed and applied on short time intervals.

The following theorem shows that the entropy of a controlled invariant subspace $V$ can be characterized by the entropy of $V$ for the corresponding uncontrolled system $\dot{x}=A x$. This result will be useful in order to compute entropy bounds.

Theorem 4.4. Let $V$ be an almost $(A, B)$-invariant subspace for system (2.1) and consider the invariance entropy $h_{\mathrm{inv}}(V)$ of control system (2.1) and the subspace entropy $h(V ; \Phi)$ of $V$ of the uncontrolled system $\Phi(t, x)=\mathrm{e}^{\text {At }} x$. Then

$$
h_{\mathrm{inv}}(V)=h(V ; \Phi) .
$$

Proof. (i) Let $K \subset V$ be compact, and fix $T, \varepsilon>0$. Consider a corresponding $(T, \varepsilon)$-spanning set $\mathcal{R}=\left\{u_{1}, \ldots, u_{\ell}\right\}$ of controls with minimal cardinality $r_{\mathrm{inv}}(T, \varepsilon)$. This means that for every $x \in K$ there is $u_{j}$ with $\operatorname{dist}\left(\varphi\left(t, x, u_{j}\right), V\right)<\varepsilon$ for all $t \in[0, T]$. By minimality, we can for every $u_{j}$ pick $x_{j} \in K$ with $\operatorname{dist}\left(\varphi\left(t, x_{j}, u_{j}\right), V\right)<\varepsilon$ for all $t \in[0, T]$. Then by linearity one finds for all $x \in K$ a control $u_{j}$ and a point $x_{j} \in K$ such that for all $t \in[0, T]$

$$
\operatorname{dist}\left(\mathrm{e}^{A t} x-\mathrm{e}^{A t} x_{j}, V\right)=\operatorname{dist}\left(\varphi\left(t, x, u_{j}\right)-\varphi\left(t, x_{j}, u_{j}\right), V\right)<2 \varepsilon .
$$

This shows that the points $x_{j}$ form a $(T, 2 \varepsilon, K, V ; \Phi)$-spanning set and hence

$$
r_{\mathrm{inv}}(T, \varepsilon, K, V) \geq r(T, 2 \varepsilon, K, V ; \Phi) .
$$

Letting $T$ tend to infinity, then $\varepsilon \rightarrow 0$ and, finally, taking the supremum over all compact subsets $K \subset V$ one obtains $h_{\text {inv }}(V) \geq h(V ; \Phi)$.

(ii) For the converse inequality, let $K$ be a compact subset of $V$ and $T, \varepsilon>0$. Let $E \subset K$ be a maximal $(T, \varepsilon)$-separated set with respect to the flow $\Phi$, say $E=\left\{y_{1}, \ldots, y_{s}\right\}$ with $s=s(T, \varepsilon, K ; \Phi)$. Then $E$ is also $(T, \varepsilon)$-spanning which means that for all $x \in K$ there is $j \in\{1, \ldots, s\}$ with

$$
\max _{t \in[0, T]} \operatorname{dist}\left(\mathrm{e}^{A t} x-\mathrm{e}^{A t} y_{j}, V\right)=\max _{t \in[0, T]} \inf _{z \in V}\left\|\mathrm{e}^{A t} x-\mathrm{e}^{A t} y_{j}-z\right\|<\varepsilon
$$


Since $V$ is almost $(A, B)$-invariant, we can assign to each $y_{j}, j \in\{1, \ldots, s\}$, a control function $u_{j} \in C\left([0, \infty), \mathbb{R}^{m}\right)$ such that $\operatorname{dist}\left(\varphi\left(t, y_{j}, u_{j}\right), V\right)<\varepsilon$ for all $t \geq 0$. Let $\mathcal{R}:=\left\{u_{1}, \ldots, u_{s}\right\} \subset C\left([0, \infty), \mathbb{R}^{m}\right)$. By linearity one has $\varphi(t, x, u)-$ $\varphi(t, y, u)=\mathrm{e}^{A t} x-\mathrm{e}^{A t} y$ for all $t \geq 0, x, y \in \mathbb{R}^{n}$ and $u \in C\left([0, \infty), \mathbb{R}^{m}\right)$. We obtain that for every $x \in K$ there is $j$ such that

$$
\max _{t \in[0, T]} \operatorname{dist}\left(\varphi\left(t, x, u_{j}\right)-\varphi\left(t, y_{j}, u_{j}\right), V\right)=\max _{t \in[0, T]} \operatorname{dist}\left(\mathrm{e}^{A t} x-\mathrm{e}^{A t} y_{j}, V\right)<\varepsilon
$$

Since $\operatorname{dist}\left(\varphi\left(t, y_{j}, u_{j}\right), V\right)<\varepsilon$ for all $t \in[0, T]$, there is $z_{1}=z_{1}(t, j) \in V$ with $\left\|\varphi\left(t, y_{j}, u_{j}\right)-z_{1}\right\|<\varepsilon$ and hence

$$
\begin{aligned}
\operatorname{dist}\left(\varphi\left(t, x, u_{j}\right), V\right) & =\inf _{z \in V}\left\|\varphi\left(t, x, u_{j}\right)-z\right\| \\
& \leq \inf _{z \in V}\left\|\varphi\left(t, x, u_{j}\right)-\varphi\left(t, y_{j}, u_{j}\right)+z_{1}-z\right\|+\left\|\varphi\left(t, y_{j}, u_{j}\right)-z_{1}\right\| \\
& <\inf _{z \in V}\left\|\varphi\left(t, x, u_{j}\right)-\varphi\left(t, y_{j}, u_{j}\right)-z\right\|+\varepsilon \\
& <2 \varepsilon .
\end{aligned}
$$

This implies that for all $x \in K$ there is $u_{j} \in \mathcal{R}$ such that

$$
\max _{t \in[0, T]} \operatorname{dist}\left(\varphi\left(t, x, u_{j}\right), V\right)<2 \varepsilon .
$$

Hence $\mathcal{R}$ is $(T, 2 \varepsilon)$-spanning and it follows that

$$
r_{\mathrm{inv}}(T, 2 \varepsilon, K, V) \leq s(T, \varepsilon, K, V ; \Phi) \text { for all } T, \varepsilon>0
$$

and consequently $h_{\text {inv }}(K, V) \leq h_{\text {sep }}(K, V ; \Phi) \leq h(V ; \Phi)$.

\section{Entropy bounds}

This section provides a general upper bound for the entropy of almost $(A, B)$-invariant subspaces of control system (2.1). Using the characterization via the subspace entropy of the associated autonomous flow, sharper upper bounds are obtained in special cases.

Theorem 5.1. (i) Let $V$ be an almost $(A, B)$-invariant subspace for system (2.1) and consider the smallest $A$-invariant subspace $\langle A \mid V\rangle$ containing $V$ and the largest $A$-invariant subspace $\mathfrak{I}^{*}(A ; V)$ contained in $V$. Then the subspace entropy of $V$ with respect to the flow $\Phi(t, x)=\mathrm{e}^{A t} x$ is finite and satisfies the inequality

$$
h(V ; \Phi) \leq \sum \max \left(0, \operatorname{Re} \lambda_{i}\right)
$$

where summation is over the eigenvalues $\lambda_{i}$ of the map induced by $A$ on the quotient space $\langle A \mid V\rangle / \mathfrak{I}^{*}(A ; V)$. (5.1).

(ii) The invariance entropy of any almost $(A, B)$-invariant subspace $V$ for system $(2.1)$ is finite and satisfies the bound

Proof. Assertion (ii) follows from (i) and Theorem 4.4. In order to prove (i) note first, that by $A$-invariance the restriction $\left.A\right|_{\langle A \mid V\rangle}$ as well as the induced map on $\langle A \mid V\rangle / \mathfrak{I}^{*}(A ; V)$ are well-defined. In order to show inequality (5.1), it suffices to show that it is satisfied for $h_{\text {span }}(K, V ; \Phi)$, where $K$ is an arbitrary compact subset of $V$. Our proof then depends on Bowen's formula for the topological entropy of linear flows, see Remark 3.2.

Consider the linear flow $\Phi(t, x)=\mathrm{e}^{A t} x, \Phi: \mathbb{R}_{0}^{+} \times \mathbb{R}^{n} \rightarrow \mathbb{R}^{n}$. By Bowen's result

$$
h_{\mathrm{top}}(\Phi)=\sum_{i=1}^{n} \max \left(0, \operatorname{Re} \lambda_{i}\right) .
$$

Analogously, the restriction of $\Phi$ to $\langle A \mid V\rangle$ has topological entropy given by the sum of the positive real parts of eigenvalues of $\left.\Phi\right|_{\langle A \mid V\rangle}$ and the induced flow $\hat{\Phi}$ on $\langle A \mid V\rangle / \mathfrak{I}^{*}(A ; V)$ has topological entropy given by the right hand side of (5.1). Hence it suffices to prove that $h(V ; \Phi) \leq h_{\mathrm{top}}(\hat{\Phi})$.

Let $K$ be a compact subset of $V$ and $\pi$ denote the projection of $\langle A \mid V\rangle$ to the quotient space $\langle A \mid V\rangle / \mathfrak{I}^{*}(A ; V)$. Thus, the set $\pi K$ is compact. Let $T, \varepsilon>0$ be given and denote by $E \subset \pi K$ a maximal $(T, \varepsilon, \pi K)$-separated set with respect to 
the flow $\hat{\Phi}$ on $\langle A \mid V\rangle / \mathfrak{I}^{*}(A ; V)$, say $E=\left\{\pi y_{1}, \ldots, \pi y_{\ell}\right\}$ with $y_{j} \in K$ and $\ell=s_{\text {top }}(T, \varepsilon, \pi K ; \hat{\Phi})$. Recall that, as noted in Remark 3.2, the topological entropy of the flow $\hat{\Phi}$ can be characterized by the exponential growth rate of $s_{\text {top }}(T, \varepsilon, \pi K ; \hat{\Phi})$. Then $E$ is also $(T, \varepsilon, \pi K)$-spanning which means that for all $x \in K$ there is $j \in\{1, \ldots, \ell\}$ with

$$
\max _{t \in[0, T]} \operatorname{dist}\left(\mathrm{e}^{A t} x-\mathrm{e}^{A t} y_{j}, \mathfrak{I}^{*}(A ; V)\right)=\max _{t \in[0, T]} \inf _{z \in \mathfrak{I}^{*}(A ; V)}\left\|\mathrm{e}^{A t} x-\mathrm{e}^{A t} y_{j}-z\right\|<\varepsilon
$$

Since $V$ is almost $(A, B)$-invariant, we can assign to each $y_{j}, j \in\{1, \ldots, \ell\}$, a control function $u_{j} \in C\left([0, \infty), \mathbb{R}^{m}\right)$ such that $\operatorname{dist}\left(\varphi\left(t, y_{j}, u_{j}\right), V\right)<\varepsilon$ for all $t \geq 0$. Let $\mathcal{R}:=\left\{u_{1}, \ldots, u_{\ell}\right\} \subset C\left([0, \infty), \mathbb{R}^{m}\right)$. By linearity one has $\varphi(t, x, u)-$ $\varphi(t, y, u)=\mathrm{e}^{A t} x-\mathrm{e}^{A t} y$ for all $t \geq 0, x, y \in \mathbb{R}^{n}$ and $u \in C\left([0, \infty), \mathbb{R}^{m}\right)$. We obtain that for every $x \in K$ there is $j$ such that

$$
\begin{aligned}
& \max _{t \in[0, T]} \operatorname{dist}\left(\varphi\left(t, x, u_{j}\right)-\varphi\left(t, y_{j}, u_{j}\right), V\right) \\
& \leq \max _{t \in[0, T]} \operatorname{dist}\left(\varphi\left(t, x, u_{j}\right)-\varphi\left(t, y_{j}, u_{j}\right), \mathfrak{I}^{*}(A ; V)\right) \\
& =\max _{t \in[0, T]} \operatorname{dist}\left(\mathrm{e}^{A t} x-\mathrm{e}^{A t} y_{j}, \mathfrak{I}^{*}(A ; V)\right) \\
& <\varepsilon .
\end{aligned}
$$

Since $\operatorname{dist}\left(\varphi\left(t, y_{j}, u_{j}\right), V\right)<\varepsilon$ for $t \in[0, T]$, there is $z_{1} \in V$ with $\left\|\varphi\left(t, y_{j}, u_{j}\right)-z_{1}\right\|<\varepsilon$ and hence, using that $V$ is a linear subspace, one finds

$$
\begin{aligned}
\operatorname{dist}\left(\varphi\left(t, x, u_{j}\right), V\right) & =\inf _{z \in V}\left\|\varphi\left(t, x, u_{j}\right)-z\right\| \\
& \leq \inf _{z \in V}\left\|\varphi\left(t, x, u_{j}\right)-\varphi\left(t, y_{j}, u_{j}\right)+z_{1}-z\right\|+\left\|\varphi\left(t, y_{j}, u_{j}\right)-z_{1}\right\| \\
& <\inf _{z \in V}\left\|\varphi\left(t, x, u_{j}\right)-\varphi\left(t, y_{j}, u_{j}\right)-z\right\|+\varepsilon \\
& <2 \varepsilon .
\end{aligned}
$$

This shows that for all $x \in K$ there is $u_{j} \in \mathcal{R} \operatorname{such}$ that $\operatorname{dist}\left(\varphi\left(t, x, u_{j}\right), V\right)<2 \varepsilon$ for all $t \in[0, T]$. Hence $\mathcal{R}$ is $(T, 2 \varepsilon, K, V)$-spanning and it follows that

$$
r_{\text {inv }}(T, 2 \varepsilon, K, V) \leq s_{\text {top }}(T, \varepsilon, \pi K ; \hat{\Phi}) \text { for all } T, \varepsilon>0
$$

Finally, taking the logarithm, dividing by $T$ and letting $T$ tend to infinity, one obtains $h_{\text {span }}(K, V ; \hat{\Phi}) \leq h_{\text {top }}(\pi K ; \hat{\Phi}) \leq$ $h_{\text {top }}(\hat{\Phi})$.

The above bound is rather conservative and can be improved in several cases. We therefore turn to the computation of sharper bounds for $h(V ; \Phi)$ under suitable genericity conditions on the almost controlled invariant subspace $V$. Here one may expect that starting in a neighborhood of the origin in $V$, only $d:=\operatorname{dim} V$ eigenvalues determine the behavior. This will be made precise below.

We begin with a few lemmas. In the sequel, $e_{1}, \cdots, e_{n}$ denotes the standard basis vectors of $\mathbb{R}^{n}$. For a real diagonalizable matrix $A$, order the eigenvalues of $A$ such that

$$
\lambda_{1} \geq \lambda_{2} \geq \ldots \geq \lambda_{n}
$$

Lemma 5.2. Let $A \in \mathbb{R}^{n \times n}$ be diagonalizable and consider a d-dimensional subspace $V \subset \mathbb{R}^{n}$ which satisfies $V \cap W=$ $\{0\}$ for any $(n-d)$-dimensional A-invariant subspace $W \subset \mathbb{R}^{n}$. We can write the Jordan representation $J$ of $A$ as

$$
J=\operatorname{diag}\left(\lambda_{1}, \ldots, \lambda_{n}\right),
$$

and we abbreviate

$$
\Lambda_{1}:=\operatorname{diag}\left(\lambda_{1}, \ldots, \lambda_{d}\right) \text { and } \Lambda_{2}:=\operatorname{diag}\left(\lambda_{d+1}, \ldots, \lambda_{n}\right) .
$$

Then there exist $S \in G L_{n}(\mathbb{R})$ and $G \in \mathbb{R}^{(n-d) \times d}$ with $V=\left\{S e_{1}, \cdots, S e_{d}\right\}$, and

$$
S^{-1} A S=\left[\begin{array}{cc}
\Lambda_{1} & 0 \\
G & \Lambda_{2}
\end{array}\right] \text {. }
$$


Proof. Let $w_{1}, \ldots, w_{n}$ be a corresponding basis of eigenvectors and denote

$$
V_{1}:=\left\langle w_{1}, \ldots, w_{d}\right\rangle \text { and } W=\left\langle w_{d+1}, \ldots, w_{n}\right\rangle \text {. }
$$

By assumption on $V$, we have $V \cap W=\{0\}$ and therefore the canonical projection map $\pi: \mathbb{R}^{n} \rightarrow V_{1}$ along $W$ maps $V$ isomorphically onto $V_{1}$. Choose any basis $v_{1}, \cdots, v_{d}$ of $V$ and extend it to a basis $S_{1}=\left(v_{1}, \cdots, v_{d}, w_{d+1}, \cdots, w_{n}\right)$. Then

$$
S_{1}^{-1} A S_{1}=\left[\begin{array}{cc}
\Gamma_{1} & 0 \\
A_{2} & \Lambda_{2}
\end{array}\right]
$$

and therefore $\Gamma_{1}$ has the same eigenvalues as $\Lambda_{1}$. Finally, we can transform $\Gamma_{1}$ to Jordan normal form by a matrix $S_{2}$. Then conjugation with the matrix

$$
S_{1} \cdot\left[\begin{array}{cc}
S_{2} & 0 \\
0 & I
\end{array}\right]
$$

leads to (5.4).

In the situation as above, by invariance of the problem under similarity, we can assume without loss of generality that

$$
A=\left[\begin{array}{cc}
\Lambda_{1} & 0 \\
G & \Lambda_{2}
\end{array}\right], V=\mathbb{R}^{d} \times\{0\}
$$

Note that for any vector $z=\left[\begin{array}{c}x \\ y\end{array}\right] \in \mathbb{R}^{n}=V \times \mathbb{R}^{n-d}$ we have $\operatorname{dist}(z, V)=\|y\|$. For $x=\left[\begin{array}{c}x_{1} \\ 0\end{array}\right], y=\left[\begin{array}{c}y_{1} \\ 0\end{array}\right] \in V$ we compute

$$
\mathrm{e}^{t A}(x-y)=\left[\begin{array}{cc}
\mathrm{e}^{t \Lambda_{1}} & 0 \\
M(t) & \mathrm{e}^{t \Lambda_{2}}
\end{array}\right]\left[\begin{array}{c}
x_{1}-y_{1} \\
0
\end{array}\right]=\left[\begin{array}{c}
\mathrm{e}^{t \Lambda_{1}}\left(x_{1}-y_{1}\right) \\
M(t)\left(x_{1}-y_{1}\right)
\end{array}\right]
$$

and

$$
\operatorname{dist}\left(\mathrm{e}^{t A}(x-y), V\right)=\left\|M(t)\left(x_{1}-y_{1}\right)\right\| .
$$

Differentiating Eq. (5.6), one finds that the $(n-d) \times d$-matrix function $M(t)$ is the unique solution to the linear differential equation $\dot{M}=\Lambda_{2} M+G \mathrm{e}^{t \Lambda_{1}}$ with initial condition $M(0)=0$ and therefore

$$
M(t)=\mathrm{e}^{t \Lambda_{2}} \int_{0}^{t} \mathrm{e}^{-s \Lambda_{2}} G \mathrm{e}^{s \Lambda_{1}} d s .
$$

The formula for $M(t)$ shows that for diagonal $\Lambda_{2}$ and $\Lambda_{1}$ one finds, with $e_{j}=j$ th standard basis vector and $g_{j}=G e_{j} \in$ $\mathbb{R}^{n-d}, j=1, \ldots, d$, for the $j$ th column of $M(t)$

$$
\begin{aligned}
M(t) e_{j} & =\mathrm{e}^{t \Lambda_{2}} \int_{0}^{t} \mathrm{e}^{-s \Lambda_{2}} G \mathrm{e}^{s \Lambda_{1}} d s e_{j}=\mathrm{e}^{t \Lambda_{2}} \int_{0}^{t} \mathrm{e}^{-s \Lambda_{2}} G \mathrm{e}^{s \lambda_{j}} e_{j} d s \\
& =\mathrm{e}^{t \Lambda_{2}} \int_{0}^{t} \mathrm{e}^{s\left(\lambda_{j} I_{n-d}-\Lambda_{2}\right)} d s g_{j} .
\end{aligned}
$$

In addition to (5.2), assume that $\lambda_{d}>\lambda_{d+1}$ and let for $k=1, \ldots, n-d, j=1, \ldots, d$

$$
\alpha_{k j}(t):=\frac{1}{\lambda_{j}-\lambda_{d+k}}\left[1-\mathrm{e}^{t\left(\lambda_{d+k}-\lambda_{j}\right)}\right], t \geq 0 .
$$

They satisfy for $t>0$ the inequalities

$$
0<\alpha_{k j}(t) \leq\left(\lambda_{j}-\lambda_{d+k}\right)^{-1} \leq\left(\lambda_{j}-\lambda_{d+1}\right)^{-1} .
$$

One computes that for $j=1, \ldots, d$

$$
M(t) e_{j}=\mathrm{e}^{t \Lambda_{2}} \operatorname{diag}\left[\alpha_{1 j}(t), \ldots, \alpha_{n-d, j}(t)\right] g_{j} .
$$


Proposition 5.3. Let $A \in \mathbb{R}^{n \times n}$ be diagonalizable and consider a d-dimensional subspace $V \subset \mathbb{R}^{n}$ which satisfies $V \cap W=\{0\}$ for any $(n-d)$-dimensional $A$-invariant subspace $W \subset \mathbb{R}^{n}$. Assume that the eigenvalues of $A$ satisfy

$$
\lambda_{1} \geq \ldots \geq \lambda_{d}>\lambda_{d+1} \geq \ldots \geq \lambda_{n} .
$$

Then the entropy $h(V ; \Phi)$ of $V$ with respect to the linear flow $\Phi(t, x)=\mathrm{e}^{A t} x$ is bounded above by the topological entropy of the flow $\Phi_{1}(t, x)=\mathrm{e}^{\Lambda_{1} t} x$ where $\Lambda_{1}=\operatorname{diag}\left[\lambda_{1}, \ldots, \lambda_{d}\right]$. Thus it satisfies the upper bound

$$
h(V ; \Phi) \leq \sum_{i=1}^{d} \max \left(0, \lambda_{i}\right) .
$$

Proof. Let $K \subset V$ be compact. We show that for $T, \varepsilon>0$ any $\left(T, \varepsilon, K ; \Phi_{1}\right)$-spanning set $R$ for the topological entropy of the flow $\Phi_{1}$ is $(T, c \varepsilon, K, V ; \Phi)$-spanning for $\Phi$, with

$$
c:=\left(\lambda_{d}-\lambda_{d+1}\right)^{-1} \max _{j=1, \ldots, d}\left\|g_{j}\right\| .
$$

Recall that the entropies do not depend on the norm, hence here and in the following we may use the 1-norm. For every $x \in K$ there is $y \in R$ such that, by formula (5.7),

$$
\begin{aligned}
\|M(t)(x-y)\|_{1} & =\left\|\sum_{j=1}^{d} M(t) e_{j}\left(x_{j}-y_{j}\right)\right\|_{1} \\
& =\left\|\sum_{j=1}^{d} \mathrm{e}^{t \Lambda_{2}} \operatorname{diag}\left[\alpha_{1 j}(t), \ldots, \alpha_{n-d, j}(t)\right] g_{j}\left(x_{j}-y_{j}\right)\right\|_{1} \\
& \leq \sum_{j=1}^{d}\left|x_{j}-y_{j}\right|\left\|\operatorname{diag}\left[\mathrm{e}^{t \lambda_{d+1}} \alpha_{1 j}(t), \ldots, \mathrm{e}^{t \lambda_{n}} \alpha_{n-d, j}(t)\right] g_{j}\right\|_{1} \\
& \leq \sum_{j=1}^{d}\left|x_{j}-y_{j}\right| \mathrm{e}^{t \lambda_{d+1}}\left(\lambda_{j}-\lambda_{d+1}\right)^{-1}\left\|g_{j}\right\|_{1} \\
& \leq\left\|\mathrm{e}^{t \Lambda_{1}}(x-y)\right\|_{1}\left(\lambda_{d}-\lambda_{d+1}\right)^{-1} \max _{j=1, \ldots, d}\left\|g_{j}\right\|_{1} \\
& \leq c \max _{t \in[0, T]}\left\|\mathrm{e}^{t \Lambda_{1}}(x-y)\right\|_{1} \\
& <c \varepsilon .
\end{aligned}
$$

This implies inequality (5.8).

Proposition 5.3 yields the following estimate for the invariance entropy of almost $(A, B)$-invariant subspaces. This estimate is sharper than the one provided in Theorem 5.1 (in particular, for low dimensional spaces $V$ ).

Theorem 5.4. Consider an almost $(A, B)$-invariant subspace $V \subset \mathbb{R}^{n}$ with dimension $d$ and denote the largest $A$ invariant subspace contained in $V$ by $\mathfrak{I}^{*}(A ; V)$ and its dimension by $\ell$. Suppose that the map $\bar{A}$ induced by $A$ on the quotient space $\mathbb{R}^{n} / \mathfrak{I}^{*}(A ; V)$ is diagonalizable and that the eigenvalues satisfy

$$
\lambda_{1} \geq \ldots \geq \lambda_{d-\ell}>\lambda_{d+1-\ell} \geq \ldots \geq \lambda_{n-\ell}
$$

Assume further that $V / \mathfrak{I}^{*}(A ; V)$ intersects trivially any $\bar{A}$-invariant subspace $W \subset \mathbb{R}^{n} / \mathfrak{I}^{*}(A ; V)$ of codimension $d-\ell$. Then the invariance entropy of $V$ satisfies the inequality

$$
h_{\text {inv }}(V) \leq \sum_{i=1}^{d-\ell} \max \left(0, \lambda_{i}\right) .
$$

Pro of. We argue similarly as in the proof of Theorem 5.1, using now the subspace entropy with respect to $V$ instead of the topological entropy. 
Let $K$ be a compact subset of $V$. Then, for the projection $\pi$ of $\mathbb{R}^{n}$ to the quotient space $\mathbb{R}^{n} / \mathfrak{I}^{*}(A ; V)$, the set $\pi K$ is compact. Let $T, \varepsilon>0$ be given and denote by $E \subset \pi K$ a maximal $(T, \varepsilon, \pi K, \pi V ; \hat{\Phi})$-separated set with respect to the flow $\hat{\Phi}$ on $\mathbb{R}^{n} / \mathfrak{I}^{*}(A ; V)$, say $E=\left\{\pi y_{1}, \ldots, \pi y_{s}\right\}$ with $y_{j} \in K$ and $s=s(T, \varepsilon, \pi K, \pi V ; \hat{\Phi})$. Then $E$ is also $(T, \varepsilon, \pi K, \pi V ; \hat{\Phi})-$ spanning which means that for all $x \in K$ there is $j \in\{1, \ldots, s\}$ with

$$
\max _{t \in[0, T]} \operatorname{dist}\left(\mathrm{e}^{A t} x-\mathrm{e}^{A t} y_{j}, V+\mathfrak{I}^{*}(A ; V)\right)=\max _{t \in[0, T]} \inf _{z \in V}\left\|\mathrm{e}^{A t} x-\mathrm{e}^{A t} y_{j}-z\right\|<\varepsilon .
$$

Since $V$ is almost $(A, B)$-invariant, we can assign to each $y_{j}, j \in\{1, \ldots, s\}$, a control function $u_{j} \in C\left([0, \infty), \mathbb{R}^{m}\right)$ such that $\operatorname{dist}\left(\varphi\left(t, y_{j}, u_{j}\right), V\right)<\varepsilon$ for all $t \geq 0$. Let $\mathcal{R}:=\left\{u_{1}, \ldots, u_{s}\right\} \subset C\left([0, \infty), \mathbb{R}^{m}\right)$. By linearity one has $\varphi(t, x, u)-$ $\varphi(t, y, u)=\mathrm{e}^{A t} x-\mathrm{e}^{A t} y$ for all $t \geq 0, x, y \in \mathbb{R}^{n}$ and $u \in C\left([0, \infty), \mathbb{R}^{m}\right)$. We obtain that for every $x \in K$ there is $j$ such that

$$
\max _{t \in[0, T]} \operatorname{dist}\left(\varphi\left(t, x, u_{j}\right)-\varphi\left(t, y_{j}, u_{j}\right), V\right)=\max _{t \in[0, T]} \operatorname{dist}\left(\mathrm{e}^{A t} x-\mathrm{e}^{A t} y_{j}, V\right)<\varepsilon
$$

Since $\operatorname{dist}\left(\varphi\left(t, y_{j}, u_{j}\right), V\right)<\varepsilon$ for $t \in[0, T]$, there is $z_{1} \in V$ with

$$
\left\|\varphi\left(t, y_{j}, u_{j}\right)-z_{1}\right\|<\varepsilon
$$

and hence, using that $V$ is a linear subspace, one finds

$$
\begin{aligned}
\operatorname{dist}\left(\varphi\left(t, x, u_{j}\right), V\right) & =\inf _{z \in V}\left\|\varphi\left(t, x, u_{j}\right)-z\right\| \\
& \leq \inf _{z \in V}\left\|\varphi\left(t, x, u_{j}\right)-\varphi\left(t, y_{j}, u_{j}\right)+z_{1}-z\right\|+\left\|\varphi\left(t, y_{j}, u_{j}\right)-z_{1}\right\| \\
& <\inf _{z \in V}\left\|\varphi\left(t, x, u_{j}\right)-\varphi\left(t, y_{j}, u_{j}\right)-z\right\|+\varepsilon \\
& <2 \varepsilon .
\end{aligned}
$$

This implies that for all $x \in K$ there is $u_{j} \in \mathcal{R}$ such that

$$
\max _{t \in[0, T]} \operatorname{dist}\left(\varphi\left(t, x, u_{j}\right), V\right)<2 \varepsilon .
$$

Hence $\mathcal{R}$ is $(T, 2 \varepsilon, K, V)$-spanning and it follows that

$$
r_{\mathrm{inv}}(T, 2 \varepsilon, K, V) \leq s(T, \varepsilon, \pi K, \pi V ; \hat{\Phi}) \text { for all } T, \varepsilon>0
$$

and consequently

$$
h_{\text {inv }}(K, V) \leq h_{\text {sep }}(\pi K, \pi V ; \hat{\Phi})=h(\pi K, \pi V ; \hat{\Phi}) \leq h(\pi V ; \hat{\Phi}) .
$$

Since, by assumption, $\pi V=V / \mathfrak{I}^{*}(A ; V)$ intersects trivially any $\bar{A}$-invariant subspace $W \subset \mathbb{R}^{n} / \mathfrak{I}^{*}(A ; V)$ of codimension $d-\ell$, it does not contain any nontrivial $\bar{A}$-invariant subspace, and we can apply Proposition 5.3 in order to prove the assertion.

We list a few explicit cases in the single-input case in which the hypotheses of Theorem 5.4 are satisfied.

Corollary 5.5. Assume that $(A, b) \in \mathbb{R}^{n \times n} \times \mathbb{R}^{n}$ is controllable and $A$ is diagonalizable with $n$ distinct real eigenvalues

$$
\lambda_{1}>\ldots>\lambda_{d}>\lambda_{d+1}>\ldots>\lambda_{n} .
$$

Let $\alpha_{1}, \cdots, \alpha_{d}$ denote any distinct real numbers that are disjoint from $\lambda_{1}, \cdots, \lambda_{n}$. Then

$$
\left.V=\operatorname{span}\left(\left(A-\alpha_{1} I\right)^{-1} b, \cdots,\left(A-\alpha_{d} I\right)^{-1} b\right)\right)
$$

is an $(A, b)$-invariant subspace with $\operatorname{ker}(A ; V)=\{0\}$ and $\langle A \mid V\rangle=\mathbb{R}^{n}$. The entropy of $V$ satisfies the inequality

$$
h_{\mathrm{inv}}(V) \leq \sum_{i=1}^{d} \max \left(0, \lambda_{i}\right)
$$


Pro of. Without loss of generality we can assume that $(A, b)$ is in Jordan canonical form, i.e. $A=\operatorname{diag}\left(\lambda_{1}, \cdots, \lambda_{n}\right)$ and $b=(1, \cdots, 1)^{\top}$. Thus $V$ coincides with the column span of the $n \times d$ matrix

$$
\left[\begin{array}{ccc}
\left(\lambda_{1}-\alpha_{1}\right)^{-1} & \cdots & \left(\lambda_{1}-\alpha_{d}\right)^{-1} \\
\vdots & & \vdots \\
\left(\lambda_{n}-\alpha_{1}\right)^{-1} & \cdots & \left(\lambda_{n}-\alpha_{d}\right)^{-1}
\end{array}\right]
$$

For any column $v$ of this matrix, the pair $(A, v)$ is controllable, which implies $\langle A \mid V\rangle=\mathbb{R}^{n}$. Let $W$ denote an arbitrary $A$-invariant eigenspace of codimension $d$ and assume $W \cap V \neq\{0\}$. Then there exists nonzero real numbers $c_{1}, \cdots c_{d}$ such that the rational function

$$
\frac{p(\lambda)}{q(\lambda)}:=\sum_{i=1}^{d} \frac{c_{i}}{\lambda-\alpha_{i}}
$$

vanishes at $d$ eigenvalues $\lambda \in\left\{\lambda_{i_{1}}, \cdots, \lambda_{i_{d}}\right\}$. But then $p=0$, as $\operatorname{deg} p<d$ and therefore $c_{1}=\cdots=c_{d}=0$, which is a contradiction. Hence $W \cap V=\{0\}$ for any $A$-invariant subspace of codimension $d$. This implies also $\operatorname{ker}(A ; V)=\{0\}$, as any $A$-invariant subspace $V_{0} \subset V$ can be extended to an $(n-d)$-dimensional $A$-invariant subspace $W$. This shows that $V$ satisfies the assumptions of Theorem 5.4.

We note that the $(A, b)$-invariant subspaces constructed in Corollary 5.5 are not of the most general form; however for $d=1$ they parameterize all one-dimensional controlled invariant subspaces. In the scalar case, it can be shown that the estimate above is sharp.

Example 5.6. Let $d=1, n=2$. We can suppose that $A$ has the form (5.5) and we use small letters instead of capital letters. Let $K \subset V=\mathbb{R} \times\{0\}$ be compact. Choose a $(T, \varepsilon, K, V ; \Phi)$-spanning set $R \subset K$. Thus for all $x \in K$ there is $y \in R$ such that for all $t \in[0, T]$

$$
\operatorname{dist}\left(\mathrm{e}^{t A}\left(\left[\begin{array}{l}
x \\
0
\end{array}\right]-\left[\begin{array}{l}
y \\
0
\end{array}\right]\right), V\right)=\|m(t)(x-y)\|<\varepsilon .
$$

If $V$ is not invariant, one has $g \neq 0$. For $t \geq 1$

$$
\begin{aligned}
\varepsilon & >\|m(t)(x-y)\|=\left\|\mathrm{e}^{t \lambda_{2}} \int_{0}^{t} \mathrm{e}^{-s \lambda_{2}} g \mathrm{e}^{s \lambda_{1}} d s(x-y)\right\| \\
& =\left\{\begin{array}{cr}
\frac{g}{\lambda_{1}-\lambda_{2}} \mathrm{e}^{t \lambda_{1}}\left[1-\mathrm{e}^{t\left(\lambda_{2}-\lambda_{1}\right)}\right]\|x-y\| & \text { for } \lambda_{1}>\lambda_{2} \\
\mathrm{e}^{t \lambda_{1}} t|g|\|x-y\| & \text { for } \lambda_{1}=\lambda_{2}
\end{array}\right. \\
& \geq c \mathrm{e}^{t \lambda_{1}}\|x-y\|,
\end{aligned}
$$

with a constant $c>0$ given by

$$
c:=\left\{\begin{array}{ccc}
\frac{|g|}{\lambda_{1}-\lambda_{2}}\left[1-\mathrm{e}^{\lambda_{2}-\lambda_{1}}\right] & \text { for } & \lambda_{1}>\lambda_{2} \\
|g| & \text { for } & \lambda_{1}=\lambda_{2}
\end{array}\right.
$$

(recall that $\lambda_{1} \geq \lambda_{2}$.) Hence

$$
\mathrm{e}^{t \lambda_{1}}\|x-y\| \leq c^{-1} \varepsilon \text { for } t \in[1, T]
$$

It is an easy observation that one also may define topological entropy by requiring the spanning property for all times $t \in[1, T]$ only. Then the set $R$ is a spanning set for the topological entropy of the flow $\mathrm{e}^{t \lambda_{1}}, t \geq 0, x \in V$. It follows that

$$
r\left(T, c^{-1} \varepsilon, K, V\right) \geq r\left(T, \varepsilon, K, \mathrm{e}^{\Lambda_{1} \cdot}\right) .
$$

Hence $h_{\text {inv }}(V) \geq h_{t o p}\left(\mathrm{e}^{\Lambda_{1} \cdot}\right)$ follows showing that equality holds in (5.11). 
Example 5.7. Here we treat the $n$-dimensional generalization of the above example, i.e. $d=1$ and $n \geq 2$. Assume further that $\lambda_{1}>\lambda_{2} \geq \cdots \geq \lambda_{n}$ and $G=\left(g_{j}\right) \in \mathbb{R}^{(n-1) \times 1}$ is nonzero. Then

$$
\|M(t)(x-y)\|=\mathrm{e}^{t \lambda_{1}}\|x-y\|\|v(t)\|
$$

with (we take the 1-norm)

$$
\|v(t)\|=\sum_{j=2}^{n}\left|g_{j}\right| \frac{1-\mathrm{e}^{t\left(\lambda_{j}-\lambda_{1}\right)}}{\lambda_{1}-\lambda_{j}}
$$

upper bounded on $[0, \infty)$ by $\bar{c}:=\sum_{j=2}^{n}\left|g_{j}\right| \frac{1}{\lambda_{1}-\lambda_{j}}$ and lower bounded on $[1, \infty)$ by $\underline{c}=\sum_{j=2}^{n}\left|g_{j}\right| \frac{1-\mathrm{e}^{\lambda_{j}-\lambda_{1}}}{\lambda_{1}-\lambda_{j}}$. Proceeding as in the above example, we conclude that the entropy is given by $\max \left(0, \lambda_{1}\right)$.

\section{Conclusions}

This paper introduces a notion of invariance entropy for controlled invariant subspaces. It essentially measures how fast the number of open-loop control functions has to grow over time, if the system is to be kept in such a subspace (more precisely, within an arbitrarily small neighborhood). If the present state of the system is completely available for the controller and hence a state feedback law can be implemented, this question is, clearly, obsolete. However, if this information is not available for the controller, the controls have to be adjusted over time and the invariance entropy gives an indication about the average number of readjustments. Thus the entropy shows the information about the state needed for a feedback; cf. also Colonius, Kawan, Nair [10] for a discussion of topological feedback entropy in a similar situation. Since the notion of controlled invariance is of fundamental importance in many feedback design problems, we hope that this will shed light on the amount of information in these problems. In particular, combining the invariance entropy discussed in the present paper with the entropy for exponential stabilization considered in Colonius [7] appears promising.

Acknowledgements Supported by DFG grants Co 124/17-1 and HE 1858/12-1 within DFG Priority Program 1305 "Control of Digitally Connected Dynamical Systems".

\section{References}

[1] F. Allgöwer and A. Zheng, (eds.), Nonlinear Model Predictive Control. Progress in Systems and Control Theory, Vol. 26, (Birkhäuser, Basel, 2000).

[2] G. Basile and G. Marro, Controlled and conditioned invariant subspaces in linear system theory, J. Optim. Theory Appl. 3, 306315 (1969).

[3] G. Basile and G. Marro, Controlled and Conditioned Invariants in Linear System Theory (Prentice Hall, Englewood Cliffs, NJ, 1992).

[4] R. Bowen, Entropy for group endomorphisms and homogeneous spaces, Trans. Am. Math. Soc. 153, 401-414 (1971).

[5] R. Carli and F. Bullo, Quantized coordination algorithms for rendezvous and deployment, SIAM J. Control Optim. 48, 1251-1274 (2009).

[6] F. Colonius, Minimal Data Rates and Invariance Entropy, Proceedings of the 19th International Symposium on Mathematical Theory of Networks and Systems - MTNS 2010, 5-9 July, 2010 (University of Budapest, Budapest, 2010).

[7] F. Colonius, Minimal bit rates and entropy for exponential stabilization, SIAM J. Control Optim. 50, 2988-3010 (2012).

[8] F. Colonius and C. Kawan, Invariance entropy for control systems, SIAM J. Control Optim. 48 1701-1721 (2009).

[9] F. Colonius and C. Kawan, Invariance entropy for outputs, Math. Control Signals Syst. 22, 203-227 (2011).

[10] F. Colonius, C. Kawan, and G. Nair, A note on topological feedback entropy and invariance entropy, Syst. Control Lett. 62, 377-381 (2013)

[11] P. A. Fuhrmann, Linear Operators and Systems in Hilbert Space (McGraw-Hill, New York, 1981).

[12] P. A. Fuhrmann and J. C. Willems, A study of $(A, B)$-invariant subspaces via polynomial models, Int. J. Control 31, 467-494 (1980).

[13] P. A. Fuhrmann and U. Helmke, On the parametrization of conditioned invariant subspaces and observer theory, Linear Algebr. Appl. 332-334, 265-353 (2001).

[14] L. Grüne and J. Pannek, Nonlinear Model Predictive Control (Springer, London, 2011).

[15] V. Gupta, A. Dana, J. Hespanha, R. Murray, and B. Hassibi, Data transmission over networks for estimation and control, IEEE Trans. Autom. Control 54, 1807-1819 (2009).

[16] A. Katok and B. Hasselblatt, Introduction to the Modern Theory of Dynamical Systems (Cambridge University Press, Cambridge, 1995).

[17] C. Kawan, Invariance entropy of control sets, SIAM J. Control Optim. 49 732-751 (2011).

[18] C. Kawan, Lower bounds for the strict invariance entropy, Nonlinearity 24, 1909-1935 (2011). 
[19] C. Kawan, Upper and lower estimates for invariance entropy, Discrete Contin. Dyn. Syst. A 30, 169-186 (2011).

[20] C. Kawan, Invariance Entropy of Control Systems, Doctoral Thesis, (Institut für Mathematik, Universität Augsburg, Augsburg, 2009).

[21] G. Marro, F. Morbidi, L. Ntogramatzidis, and D. Prattichizzo, Geometric Control Theory for Linear Systems: A Tutorial, Proc. of the 19th International Symposium on Mathematical Theory of Networks and Systems (MTNS), 5-9 July, 2010, Budapest, Hungary (University of Budapest, Budapest, 2010).

[22] A. S. Matveev and A. V. Savkin, Estimation and Control Over Communication Networks (Birkhäuser, Basel, 2009).

[23] G. N. Nair, R. J. Evans, I. M. Y. Mareels, and W. Moran, Topological feedback entropy and nonlinear stabilization, IEEE Trans. Autom. Control, 49(9), 1585-1597 (2004).

[24] M. Pollicott and M. Yuri, Dynamical Systems and Ergodic Theory (Cambridge University Press, Cambridge, 1998).

[25] C. Robinson, Dynamical Systems. Stability, Symbolic Dynamics, and Chaos, Second edition. (CRC Press, Boca Raton, 1999).

[26] H. L. Trentelman, Almost Invariant Subspaces and High Gain Feedback, PhD Thesis (Rijksuniversiteit Groningen, Groningen, 1985).

[27] H. L. Trentelman, A. A. Stoorvogel, and M. Hautus, Control Theory for Linear Systems (Springer, Berlin, Heidelberg, New York, 2001).

[28] J. Trumpf, On the Geometry and Parametrization of Almost Invariant Subspaces and Observer Theory, Doctoral Thesis (Institut für Mathematik, Universität Würzburg, Würzburg, 2002).

[29] P. Walters, An Introduction to Ergodic Theory (Springer, Berlin, Heidleberg, New York, 1982).

[30] J. C. Willems, Almost $A(\bmod B)$-invariant subspaces, Asterisque 75-76, (1980), pp. 239-248.

[31] J.C. Willems, Almost invariant subspaces: An approach to high gain feedback design - Part I: Almost controlled invariant subspaces, IEEE Trans. Autom. Control 26(1), 235-252 (1981).

[32] M. W. Wonham, Linear Multivariable Control: A Geometric Approach (Springer, Berlin, Heidelberg, New York, 1979).

[33] W. Wong and R. Brockett, Systems with finite communication bandwidth constraints. I. State estimation problems, IEEE Trans. Autom. Control 42, 1294-1299 (1997).

[34] W. Wong and R. Brockett, Systems with finite communication bandwidth constraints. II. Stabilization with limited information feedback, IEEE Trans. Autom. Control 44, 1049-1053 (1999). 